\title{
GAMBOGIC ACID AS ANTICANCER AGENT: A REVIEW
}

Syed Amir Gilani ${ }^{1}$, Muhammad Imran ${ }^{1}$, Iahtisham-ul-Haq ${ }^{2}$, Riffat Mehboob ${ }^{1}$, Wafa Fatima ${ }^{3}$, Amber Hassan ${ }^{1}$

${ }^{1}$ University Institute of Diet and Nutritional Sciences, Faculty of Allied Health Sciences, The University of Lahore-Lahore, Pakistan

${ }^{2}$ National Institute of Food Science and Technology, University of Agriculture Faisalabad, Pakistan

${ }^{3}$ University Institute of Public Health, Faculty of Allied Health Sciences, The University of Lahore, Lahore, Pakistan

\begin{abstract}
Gambogic acid, a common traditional Chinese medicine and widely distributed throughout South China, Vietnam, Cambodia, and Thailand. It is prenylated xanthone which is the significant bioactive compound of gamboge. Gambogic acid is known as a strong apoptotic inducer in cancer cells. It has been found as strong anticancer agent against various types of cancer cells lines such as breast cancer, pancreatic, and cervical cancer. It induces apoptosis, down regulates the antiapoptotic proteins (survivin and BCL2,) and down regulates the activities of P-glycoprotein in drug sensitive human breast MCF-7 and drug-resistant MCF-7/ADR cells. Similarly, it also exerts alteration in P13K, AKT, p21, MMP-2 \&-9, and phosphorylated-AKT expressions. The current review highlights the anticancer and chemo-preventive perspectives of gambogic acid and its mechanistic role against human and animal cancers.
\end{abstract}

Key words: Gambogic acid, phytochemical, breast cancer, pancreatic cancer, glioma cancer

\section{Introduction}

Natural bioactive compounds from fruits and vegetables have been used as therapeutic agents in clinical and biological activity against various human maladies such as cancer, cardiovascular, and neurodegenerative disorders. Gambogic acid (GA), a naturally occurring compound which is derived from Garcinia hanburyi tree. It has been found to exert multiple intracellular and extracellular actions such as antimetastatic, anti-inflammatory autophagy, programmed cell death, anti-angiogenesis, and cell cycle arrest, respectively (Kashyap et al., 2016; Seo et al., 2019). Multiple molecular targets and pathways of gambogic acid have been studied such as caspase-3 apoptosis, PI3K/Akt, TNF- $\alpha$, ATR-Chk1, and MET pathways (Ishaq et al., 2014). Likewise, GA 
induces apoptotic cell death via mitochondrial pathway, enhancing the active oxygen concentration, suppressing the inhibiting the NF- $\kappa \mathrm{B}, \mathrm{MAPK} / \mathrm{ERK}$, and PI3K/AKT expressions in HT-29 cells (Lü et al., 2012; Wang et al., 2014). It also suppresses the cancer cell proliferation and cell growth in various human cancers i.e. breast, prostate, pancreatic, colon, cervical and lung etc (Kashyap et al., 2016).

\section{Anticancer perspectives}

\section{Breast cancer}

GA has been found to prevent from tumorigenesis in the MCF-7 subcutaneous xenograft tumor model through inhibiting proliferation and inducing apoptosis (Wang et al., 2018a; Sang et al., 2018). Tumor necrosis factor related apoptosis- inducing ligand (TRAIL) has the ability to induce apoptosis in cancer cells that has developed significant interest in treating different types of cancers. In breast cancer cells, gambogic acid increased sensitivity of TRAIL and increased TRAIL-induced apoptosis in cancer cells. Wang et al (2018) discovered that GA with TRAIL cooperation decreased anti-apoptotic proteins level and activated Bid (BH3 interacting-domain death agonist) which promoted cross talk between extrinsic and intrinsic apoptotic signaling, instead of enhancing the TRAIL DR4 and DR5 receptors (Wang et al., 2018b). Different researchers and investigators determined that encapsulation of GA with polyethylenimine-poly (d,1-lactide-coglycolide) caused momentous augmentation in apoptotic cell death in an in vitro study on triplenegative breast cancer, and in vivo suppression of TNBC tumor growth (Wang et al., 2017; Xu et al., 2016). In another exploration by Doddapaneni et al. 2016, they found that encapsulation of GA as PEGylated liposomal formulation in MDA-MB-231 orthotopic xenograft significantly suppressed the growth of tumor, with 50\% tumor-volume, and reduced tumor weight alongside inhibition of Bcl2 expression, apoptotic markers, cyclinD1, and micro-vessel density markerCD31. Moreover, it also increased the levels of p53 and Bax (Doddapaneni et al., 2016).

GA increased cell toxicity, apoptosis, down regulated expressions of anti-apoptotic proteins survivin and $\mathrm{Bcl} 2$, and downregulated expression and activities of P-glycoprotein (P-gp) in drug sensitive human breast MCF-7 and drug-resistant MCF-7/ADR cells (Wang et al., 2015b). Sensitization of doxorubicin (DOX) resistant breast cancer cells to DOX-mediated cell death and enhancement in the intra-cellular accumulation of DOX via inhibiting both P-gp expression and activity were reported after gambogic acid treatment. In addition, DOX in combination with GA 
led to the production of intra-cellular reactive oxygen species and inhibition of anti-apoptotic protein surviving. In DOX-induced apoptosis, over-expression of surviving blocked the sensitizing effects of GA. Moreover, ROS-mediated activation of p38 MAPK was revealed in GA -mediated suppression of Survivin expressions (Wang et al., 2015a).

\section{Glioma cancer}

A group of researchers studied the anticancer role of GA against human U251 glioma cells, they found that reduction in phosphorylation of P38, AKT, and mTOR, as well as decrement in the upstream binding factor (UBF), phosphorylation of ribosomal protein precursors (Pre) and insulinlike growth factor I (IGF-1) were reported after GA treatment (Luo et al., 2020; Sang et al., 2019). In T98G glioblastoma cells, GA dose dependently showed potent anti-proliferative activity via apoptosis induction, enhancement in Bax and AIF expression, PARP and cleavage of caspase-3,$7 \&-9$ and down regulation of Bcl-2 expression (Thida et al., 2016). A peer of researchers, they investigated induction of up regulation of leucine-rich repeat and Ig-like domain-containing1(LRIG1) which further exhibited downstream Akt/mTORC1 inhibition and epidermal growth factor receptor (EFGR) degradation after GA treatment in U87 glioma cells. Further, U87 cell apoptosis and growth inhibition, AMP-activated protein kinase activation mediated GA-induced LRIG1 upregulation, while AMPK inhibition by shRNA or compound C reduced GA -induced EGFR/Akt inhibition and cytotoxicity in U87 cells (He et al., 2013). Time and dose- dependent anti-cancer action of GA investigated by group of researchers against human glioma cell lines such as U251MG and U87MG showed decreased cell proliferation, induced apoptosis in cells, and inhibited colony formation. Development of monodansylcadaverine in autophagic vacuoles, up regulation of expressions of Beclin 1, LC3-II and Atg5, enhancement in punctate fluorescent signals of glioblastoma cells pre-transfected with GFP-tagged LC3 plasmid were reported (Luo et al., 2012). During an in vitro study on rat C6 glioma cells, GA dose and time dependently induced apoptotic cell death as it triggered intrinsic mitochondrial apoptotic pathways whereas during an in vivo trial, intravenous treatment of GA showed momentous reduction in tumor volumes through apoptotic induction (Qiang et al., 2008).

\section{Skin cancer}

In recent study reported by Li and their colleagues, they found that dose and time dependent GA in vitro study in melanoma A375, B16-F10 cells regulating the protein expressions, inhibiting the 
proliferation, migration, invasive and adhesive properties, suppressing the EMT and angiogenesis processes and reducing the enzymatic activities of MMP-2 and MMP-9. Furthermore, GA also suppressed the abnormal PI3K/Akt and ERK signaling pathways (Li et al., 2019). In human malignant melanoma (MM) cells A375, GA (2.5-7.5 microg/mL) for $36 \mathrm{~h}$ suppressed the caspase3 activity and $\mathrm{Bax} / \mathrm{Bcl}-2$ ratio, respectively (Xu et al., 2009).

\section{Types Mechanisms \\ Breast cancer Inhibited proliferation and induced apoptotic cell death}

Glioma cancer

Skin cancer

Colon cancer

Liver cancer

Lung cancer (TNF)-related apoptosis-inducing ligand (TRAIL)

Enhanced TRAIL-induced apoptosis

Caused momentous augmentation in apoptotic cell death

Suppressed TNBC tumor growth reduction in tumor weight p53 and Bax (IGF-1) cleavage of caspase-3, $-8,-9$, and PARP

Down regulated Bcl-2 expression Inhibited downstream Akt/mTORC1 properties

Inhibited the EMT and angiogenesis processes

Induced cell proliferation, migration and invasion phosphorylated-AKT

Enhanced the levels of p-AMPK

Up-regulated E-cadherin linked with LKB1

Induced E-cadherin, and down regulated the ZEB1
Increased the sensitivity of breast cancer cells to tumor necrosis factor

Significantly suppressed the tumor growth, tumor volume, and caused

Showed inhibition on expression of bcl2, apoptotic markers, surviving, cyclinD1, and microvessel density marker-CD31. Increased the levels of

Caused reduction in phosphorylation of P38, AKT, and mTOR

Lowered the upstream binding factor (UBF), phosphorylation of ribosomal protein precursors (Pre) and insulin-like growth factor I

Induced apoptosis and increased Bax and AIF expression, Enhanced

Suppressed the epidermal growth factor receptor (EFGR) degradation

Suppressed the proliferation, migration, invasive and adhesive

Lowered the enzymatic activities of MMP-2 and MMP-9 Suppressed the abnormal PI3K/Akt and ERK signaling pathways.

Altered expressions of AKT, p21, P13K, MMP-2 and -9, and

Suppressed the cell viability and activated the SHH signaling

Suppressed the antioxidant enzyme TrxR1 reduction activity

Enhanced intracellular ROS level, C/EBP-homologous protein,

expression levels of glucose-regulated protein (GRP)78

Activated transcription factor 6 and caspase 12

Activated the inositol-requiring enzyme 1alpha and phosphorylation

levels of protein kinase R-like ER kinase

Inhibited cell growth, induced cells autophagy

Caused upregulation of Beclin 1, and transformation of autophagosome markers such as LC31 to LC2II

\section{References}

(Wang et al.,2018a; Sang et al., 2018).

(Wang et al., 2018b).

(Wang et al., 2017;Xu et al., 2016)

(Doddapaneni et al., 2016)

(Luo et al., 2020;Sang et al., 2019)

(Thida et al., 2016)

(He et al., 2013).

(Li et al., 2019)

(Zhou and Ma, 2019).

(Wang et al., 2019).

(Li et al., 2019;Kebebe et al.,

2019; Yin et al., 2014).

(Duan et al., 2014).

(Zhu et al., 2019).

(Ye et al., 2018). 
Lowered the expression levels of Jagged1, Jagged2, DLL1, DLL3, DLL4, PK3K and Bcl2

(Zhu et al., 2018)

Enhanced expression level of active caspase 3

Inhibited Akt phosphorylation, and NICD nuclear translocation

Pancreatic Induced the expression of Beclin-1 and LC3-II proteins

(Wang et al., 2019).

cancer

Destructed mitochondrial membrane

Down regulated the TOP2A, ALDOA, and ATG4B

Up-regulated DUSP1, DDIT3, and DUSP5

Blood cancer

Enhanced the efficacy rate

(Youns et al., 2018)

Inhibited cell proliferation

Down regulated the levels of nuclear factor- $\kappa \mathrm{B}(\mathrm{NF}-\kappa \mathrm{B}), \mathrm{BCL}-2$,

(Feng et al., 2018).

phosphatidylinositol3-kinase (PI3K), c-myc, and phosphorylation of

serine-threonine kinase ( $\mathrm{p}-\mathrm{AKT})$

Table 1: Role of Gambogic acid in different cancers

\section{Colon cancer}

Zhou and Ma recently reported anti-tumor activity of GA in humans against colon cancer cells SW620 cells at $10 \mu \mathrm{g} / \mathrm{ml}, 50 \mu \mathrm{g} / \mathrm{ml}$ and $100 \mu \mathrm{g} / \mathrm{mL}$ with $10 \mu \mathrm{g} / \mathrm{ml} 5$-fluorouracil. They concluded that GA induced cell proliferation, migration and invasion of cancer cells dose dependently and it also altered expressions of AKT, p21, P13K, MMP-2 \&-9, and phosphorylated-AKT (Zhou and Ma, 2019). In another animal study conducted by Wang and the colleagues, explored that GA enhanced the apoptosis in cancer cells along with suppression of cell viability and activated the sonic hedgehog (SHH) signaling (Wang et al., 2019). Different doses of GA $(5,10$ and $20 \mathrm{mg} / \mathrm{kg}$ in saline) against HT-29 cells of BALB/c nude mice twice a week inhibited cell proliferation through apoptosis and mediated by intrinsic (mitochondrial) and extrinsic (death receptors) pathways in a dose dependent manner (Huang et al., 2015a). Nano-encapsulated GA studied by Huang and coworkers found that GA against HT-29 human colon cancer xenograft mouse models produced anticancer role by decreasing cell division and proliferation and inducing apoptosis (Huang et al., 2015b). GA in LOVO cells also significantly induced apoptosis and inhibited the proliferation in a dose- and time-dependent manner. It has been reported that magnetic nano-particles of GA containing $\mathrm{Fe}_{3} \mathrm{O}_{4}$ have more ability to increase transcription of cytochrome c, caspase 9, and caspase 3 genes and decreased the transcription of phosphatidylinositol 3-kinase, Akt, and Bad genes as compare to the gambogic acid without magnetic nanoparticles containing $\mathrm{Fe}_{3} \mathrm{O}_{4}$. $(\mathrm{Fang}$ et al., 2012; Wei et al., 2018). Combined form of GA with 5-fluorouracil (5-FU) treatment against HCT116 human CRC cells, SW480 and human intestinal epithelial cells (IECs) significantly 
inhibited growth of cancer cells, apoptosis and decreased P53, survivin and TS mRNA and protein levels as well as increased P53 protein levels (Wei et al., 2017; Zhang et al., 2017). Likewise, in both 5-fluorouracil (5-FU) sensitive and 5-FU resistant colorectal cancer cells, GA suppressed the proliferation and induced apoptosis via activating JNK signaling pathway (Wen et al., 2015; Zhang et al., 2014).

\section{Liver cancer}

Liver kinase-B1 (LKB1) have tumor suppressing action by regulating the cell growth, survival, metabolism, and polarity. GA has been found to enhance the levels of p-AMPK via up-regulating E-cadherin linked with LKB1 in vivo and in vitro studies. Additionally, GA also induces Ecadherin, and down regulates the ZEB1 (Li et al., 2019;Kebebe et al., 2019; Yin et al., 2014). The TrxR1 (thioredoxin reductase (TrxR) isoenzymes,1) in nucleus or cytosol and TrxR2 in mitochondria are important mammalian selenocysteine ( $\mathrm{Sec}$ )-containing flavor-enzymes with a Cterminal-Gly-Cys-Sec-Gly active site are frequently over-expressed in human tumor and GA reduced expression of these malignant cells which reverse tumor growth. Duan et al. in 2014 studied that GA against hepatocellular carcinoma SMMC-7721 cells targeted mammalian selenocysteine ( $\mathrm{Sec}$ )-containing flavoenzymes residue in the antioxidant enzyme TrxR1 to inhibit its Trx-reduction activity which collapsed intracellular redox balance and leads to the accumulation of reactive oxygen species. Furthermore, over-expression of TrxR1 in cells decreased GA induced cytotoxicity while knockdown of TrxR1 sensitizes cells to GA (Duan et al., 2014). In hepato-cellular carcinoma liver cell line (HepG2) cells, GA and cadmium-tellurium quantum dots enhanced accumulation of drugs and decreased cell proliferation. Nano-composition of GA -CdTe enhanced drug action of gambogic acid molecules against HepG2 cells and induced cell cycle arrest at G2/M phase along with cell apoptosis (Xu et al., 2013). GA inhibited Huh7 and Hep3B growth in same apoptotic pathways and $\mathrm{IC}_{50}$ investigated for both cell lines were 1.8 and 2.2 $\mu \mathrm{M}$, respectively (Lee and Ho, 2013).

GA has antitumor effects against hepatocellular carcinoma through multiple mechanisms such as (i) improvement in liver functions, (ii) reduction of cell lesions in hepatic tissue, (iii) suppression of expression of CD105, and (iv) prolongation of survival time, respectively (Yan et al., 2017). In hepatocellular carcinoma (HCC) tissues, gambogic acid inhibited UNC119 (uncoordinated 119 or retinal protein 4) by inhibiting cell proliferation, produced cell cycle arrest at G0 and G1 phases 
against Hep3B cells, down regulated cell cycle related proteins including cyclin A, E, D1 and cyclin-dependent kinase 2, 4, 6. GA also down-streamed UNC119 and decreased glycogen synthase kinase $3 \beta(\mathrm{Gsk} 3 \beta) / \beta$-catenin signaling (Wu et al., 2016).

A study reported by Deschatrette et al. (2013) showed that gambogic acid i has been found to lower the thymidylate synthetase mRNA levels and level of thymidylate synthetase mRNA $n$ a gastric carcinoma cell line. A contradictive relationship was observed in hepatoma cells between DHFR expression and resistance to GA with a dihydrofolate reductase (DHFR) gene amplification and cells transfected with an inducible DHFR transgene. In vitro, GA suppressed the DHFR activity, and lowered the affinity of the enzyme for dihydrofolate (Deschatrette et al., 2013). In an in vitro study human hepatocellular carcinoma (HCC) cells cytotoxicity i.e. Bel-7402 cells and HepG2 cells by GA inhibited cell proliferation, and induced apoptosis. GA might become a promising therapeutic agent for the treatment human HCC (He et al., 2012). There are multiple pathways involved in HepG2 cells through the application of GA in a concentration-dependent manner such as inhibition of viability of HepG2 cells, reduction in volume, condensation of chromatin, enhancement in apoptotic cells percentage, alteration of expression level of several apoptosisassociated proteins, Bcl-2, p53, Bax and pro-caspase-3. In addition, GA exhibited apoptosis in HepG2 cells, probably via intrinsic mitochondrial pathways (Mu et al., 2010).

A study reported by Nie and the colleagues determined that GA destroyed mitochondrial membrane potential and induced accumulation of ROS dose dependently in human hepatoma SMMC-7721 cells with the release of cytochrome $\mathrm{c}$ and apoptosis-inducing factor from mitochondria to cytosol, which induced apoptosis in the cells. Furthermore, it was also concluded that GA improved the level of the phosphorylation of c-jun-N-terminal protein kinase (JNK) and p38 (Nie et al., 2009). In china gamboge is developed and used as an injectable drug against cancer treatment. Wang et al. in 2009 investigated inhibition ratio and IC $_{50}$ values of two derivatives of gamboge (gambogic acid and gambogenic acid) against HCC and revealed its inhibitory effects on cell proliferation. Both compounds up regulated the guanine nucleotidebinding protein beta subunit 1, and cyclin-dependent kinase 4 inhibitor A whereas down regulated the stathmin 1 (STMN1) and 14-3-3 protein sigma expressions. Furthermore, overexpression of STMN1 in HCC cells decreased their sensitivity, whilst small interfering RNAs targeting STMN1 enhanced their sensitivity to GA and gambogenic acid (Wang et al., 2009). 


\section{Lung cancer}

Different doses of GA as $0,0.5$, and $1.0 \mu \mathrm{mol} / \mathrm{L}$ were treated with non-small cell lung cancer A549 cells in a concentration-dependent manner and reduced the cell viability, enhancement intracellular ROS level, C/EBP-homologous protein, expression levels of glucose-regulated protein (GRP)78, activated transcription factor 6 and caspase 12, as well as the inositol-requiring enzyme 1 alpha and phosphorylation levels of protein kinase R-like ER kinase (Zhu et al., 2019). A study conducted by $\mathrm{Ye}$ and co-workers, they explored that gambogic acid in non-small cell lung cancer (NSCLC) NCI-H441 of xenografts inhibited cell growth, induced cells autophagy, caused upregulation of Beclin 1, and transformation of autophagosome markers such as LC31 to LC2II. Moreover, GA induced autophagy through an ROS-dependent pathway (Ye et al., 2018). In human non-small cell lung cancer (NSCLC) cells (A549 and SPC-A1 cells), different doses of GA at $0,0.5,0.75$, and $1.0 \mu \mathrm{mol} / 1$ showed momentous inhibition in cell viability, enhancement in cell apoptosis, reduction in the expression levels of Jagged1, Jagged2, DLL1, DLL3, DLL4, PK3K and Bcl2, increment in expression level of active caspase-3, and inhibited Akt phosphorylation, and NICD nuclear translocation (Zhu et al., 2018). Combined form of GA with cisplatin produced a significant inhibitory effect on A549 and NCI-H460 cells. They significantly increased autophagy, and inhibited the activation of S6, mTOR and Akt. In addition, gambogic acid combined with rapamycin induced more cell death as compare to GA anti-tumor action alone (Zhao et al., 2017).

\section{Pancreatic cancer}

In a recent study, pancreatic cancer cell lines treated with gambogic acid and showed induction of the expression of Beclin-1 and LC3-II proteins and enhancement in the formation of both acidic vesicular organelles and autophagosomes, as well as autophagic flux. Moreover, increased cytotoxicity of gambogic acid was linked with inhibition of autophagy by chloroquine. In addition, promotion of ROS production and destruction of mitochondrial membrane which is linked with autophagy activation were reported after GA treatment (Wang et al., 2019).

Pancreatic cancer cells treated with GA showed growth inhibition, down-regulation of TOP2A, ALDOA, and ATG4B, and up-regulation of DUSP1, DDIT3, and DUSP5, respectively (Youns et al., 2018). Similarly, there are multiple mechanisms through gambogic acid treatment against pancreatic cancer cell lines such as suppression of the growth, and induction of cell cycle arrest at 
S-phase and apoptosis. In PANC-1 and BxPC-3 cells, synergistic action of GA with gemcitabine was reported. GA activated apoptosis induced by gemcitabine via increasing expression of cleaved caspase-3 and 9, cleaved-PARP and Bax and reduced Bl-2 expressions. Reduction in the expression of the ribonucleotide reductase subunit-M2 (RRM2) protein and mRNA were reported after GA treatment which is linked with resistance to gemcitabine via extracellular signal regulated kinase $(\mathrm{ERK}) / \mathrm{E} 2 \mathrm{~F} 1$ signaling pathway inhibition. Furthermore, both compounds in the xenograft cancer model significantly repressed tumor growth and down regulated the p-ERK, E2F1, and RRM2 (Xia et al., 2017;Saeed et al., 2014). In Panc-1 pancreatic cancer cells, gambogic acidloaded magnetic $\mathrm{Fe}_{3} \mathrm{O}_{4}$ nanoparticles significantly inhibited the ETS1-mediated proliferation and migration of cells, lowered the expression of ETS1, as well as it down-streamed target genes cyclin D1, u-PA and VEGF (Wang et al., 2012). In another study, encapsulation of GA with magnetic $\mathrm{Fe}_{3} \mathrm{O}_{4}$ nanoparticles improved anticancer potential via induction of apoptosis, increasing of Bax, caspase 3 and caspase 9, decreasing protein expressions of Bcl-2 (Wang et al., 2011).

\section{Blood cancer}

GA increased production of ROS in hematopoietic malignant cell lines (Ortiz-Sánchez et al., 2009). In acute myeloid leukemia cell lines of experimental subjects such as Jurket, HL-60 and MV4-11 cells, nano-emulsion encapsulated GA enhanced the efficacy rate in both in vivo and in vitro studies (Feng et al., 2018). Administration of gambogic acid more than $0.5 \mu \mathrm{M}$ to human leukemia cell line K562 dose-dependently induced apoptosis, inhibited cell proliferation, and down regulated the levels of nuclear factor- $\kappa \mathrm{B}(\mathrm{NF}-\kappa \mathrm{B}), \mathrm{BCL}-2$, phosphatidylinositol3-kinase (PI3K), c-myc, and phosphorylation of serine-threonine kinase (p-AKT) (Chen et al., 2015).

Cancer cells lines such as U937 and HL-60 were treated with gambogic acid and caused suppression in cell growth, promotion in differentiation, and upregulation of p21waf1/cip1 expression, respectively (Chen et al., 2014). Gambogic acid also reported as significant anti-cancer agent against different chronic myelogenous leukemia cell lines i.e. KBM5-T315I, KBM5, and K562 of nude mice via inducing apoptosis, cell proliferation inhibition and suppression of the growth of imatinib-resistant Bcr-Abl-T315I (Shi et al., 2014). In another study reported by Li and colleagues, they found that down regulation in the expression of SRC-3 along with inhibition of Akt kinase and its down-streamed targets p70, S6 kinase 1(S6K1) and glycogen synthase kinase 3 beta (GSK3beta) were observed after gambogic acid treatment against myelogenous leukemia cell 
line K562 cells in humans. In addition, these changes also affected the expressions of apoptosis (gene Bcl-2) in K562 cells (Li et al., 2009).

In another study conducted by Tao and colleagues, they explored that N-(2-ethoxyethyl) gambogamide (NG-18), a derivative of gambogic acid in against leukemia (HL-60 cells) also effectively suppressed the culture human tumor cells proliferation, induced apoptosis whereas tumor apoptosis is linked with up-regulated pro-apoptotic Bcl-2 family member Bax, and downregulated anti-apoptotic protein Bcl-2 (Tao et al., 2007). In multidrugresistant lymphoma Raji/DNR cells, gambogic acid in a dose-dependent manner down-regulated expression of P-glycoprotein (Zhou et al., 2016). A study reported by Zhao and co-workers showed that gambogic acid exhibited mechanisms such as suppression of cell growth, induction of apoptosis, caused over expression of SRC-3, suppressed cyclin D3 and Bcl-2, Bcl-6 expressions, modulated down-stream gene expression, and induced the de-acetylation of histone $\mathrm{H} 3$ at lysine 9 and lysine 27 in B-cells non-Hodgkin lymphoma (NHL) (Zhao et al., 2016). Likewise, a group of researchers unveiled that GA in vitro and in vivo trials has potent anticancer effect on Bcell lymphoma (DLBCL) cells such as GCB- and ABC-DLBCL cells through inducing apoptosis, and inhibiting cells growth (Shi et al., 2015). In a study against Jeko-1 human mental cells lymphoma cell apoptosis, GA inhibited cell growth as time and dose-dependently. It is involved in suppressing ratio of Bax and $\mathrm{Bcl}-2$ with arresting cell cycle and decreasing mitochondrial membrane potential and activating caspase-3,-8,-9 (Xu et al., 2013).

\section{Conclusion}

Researchers conducted extensive work on natural products to explore their role as an anticancer agent in last decades. Among these products, GA has attained popularity as a promising novel antitumor agent and has ability to inhibit proliferation and induce apoptosis. It inhibits the cell viability, enhances cell apoptosis, reduces the expression levels of PK3K, DLL1, DLL3, DLL4, Jagged1, Jagged2, and Bcl2, increases the active caspase-3 level, and inhibits the Akt phosphorylation in human malignancies. Extensive work is required to further investigate its toxicity and interaction with other chemotherapic agents in clinical setting.

\section{References}


Chen J, Zhou M, Zhang Q, Xu J, Ouyang J. Anticancer Effect and Apoptosis Induction of Gambogic Acid in Human Leukemia Cell Line K562 In Vitro. Med Sci Monit. 2015 Jun 2;21:1604-10. doi: 10.12659/MSM.893004.

Chen Y, Hui H, Li Z, Wang HM, You QD, Lu N. Gambogic acid induces growth inhibition and differentiation via upregulation of p21waf1/cip1 expression in acute myeloid leukemia cells. J Asian Nat Prod Res. 2014 Oct;16(10):1000-8. doi: 10.1080/10286020.2014.918108. Epub 2014 May 19.

Deschatrette J, Ng-Bonaventure K, Philippe L, Wolfrom C. Interaction between Gambogic acid and dihydrofolate reductase and synergistic lethal effects with methotrexate on hepatoma cells. Anticancer Res. 2013 Jan;33(1):133-42.

Doddapaneni R, Patel K, Owaid IH, Singh M. Tumor neovasculature-targeted cationic PEGylated liposomes of gambogic acid for the treatment of triple-negative breast cancer. Drug Deliv. 2016 May;23(4):1232-41. doi: 10.3109/10717544.2015.1124472. Epub 2015 Dec 24.

Duan D, Zhang B, Yao J, Liu Y, Sun J, Ge C, Peng S, Fang J. Gambogic acid induces apoptosis in hepatocellular carcinoma SMMC-7721 cells by targeting cytosolic thioredoxin reductase. Free Radic Biol Med. 2014 Apr;69:15-25. doi: 10.1016/j.freeradbiomed.2013.12.027. Epub 2014 Jan 7.

Fang L, Chen B, Liu S, Wang R, Hu S, Xia G, Tian Y, Cai X. Synergistic effect of a combination of nanoparticulate $\mathrm{Fe} 3 \mathrm{O} 4$ and gambogic acid on phosphatidylinositol 3-kinase/Akt/Bad pathway of LOVO cells. Int J Nanomedicine. 2012;7:4109-18. doi: 10.2147/IJN.S32475. Epub 2012 Jul 30. Feng Z, Wang Z, Yang Y, Du Y, Cui S, Zhang Y, Tong Y, Song Z, Zeng H, Zou Q, Peng L, Sun H. Development of a safety and efficacy nanoemulsion delivery system encapsulated gambogic acid for acute myeloid leukemia in vitro and in vivo. Eur J Pharm Sci. 2018 Dec 1;125:172-180. doi: 10.1016/j.ejps.2018.10.001. Epub 2018 Oct 5.

He L, Ling Y, Fu L, Yin D, Wang X, Zhang Y. Synthesis and biological evaluation of novel derivatives of gambogic acid as anti-hepatocellular carcinoma agents. Bioorg Med Chem Lett. 2012 Jan 1;22(1):289-92. doi: 10.1016/j.bmcl.2011.11.016. Epub 2011 Nov 11.

He XY, Liu XJ, Chen X, Bian LG, Zhao WG, Shen JK, Sun QF. Gambogic acid induces EGFR degradation and Akt/mTORC1 inhibition through AMPK dependent-LRIG1 upregulation in cultured U87 glioma cells. Biochem Biophys Res Commun. 2013 Jun 7;435(3):397-402. doi: 10.1016/j.bbrc.2013.04.099. Epub 2013 May 9. 
Huang GM, Sun Y, Ge X, Wan X, Li CB. Gambogic acid induces apoptosis and inhibits colorectal tumor growth via mitochondrial pathways. World J Gastroenterol. 2015a. May 28;21(20):6194205. doi: 10.3748/wjg.v21.i20.6194.

Huang W, Wang X, Shi C, Guo D, Xu G, Wang L, Bodman A, Luo J. Fine-tuning vitamin Econtaining telodendrimers for efficient delivery of gambogic acid in colon cancer treatment. Mol Pharm. 2015b. Apr 6;12(4):1216-29. doi: 10.1021/acs.molpharmaceut.5b00051. Epub 2015 Mar 2.

Ishaq M, Khan MA, Sharma K, Sharma G, Dutta RK, Majumdar S. Gambogic acid induced oxidative stress dependent caspase activation regulates both apoptosis and autophagy by targeting various key molecules (NF- $\mathrm{B}$, Beclin-1, p62 and NBR1) in human bladder cancer cells. Biochim Biophys Acta. 2014;1840:3374-3384. doi: 10.1016/j.bbagen.2014.08.019.

Kashyap, D., Mondal, R., Tuli, H.S., Kumar, G., Sharma, A.K.2016. Molecular Targets of Gambogic Acid in Cancer: Recent Trends and Advancements. Tumour Biol. 2016 Oct;37(10):12915-12925. doi: 10.1007/s13277-016-5194-8.

Kebebe D, Wu Y, Zhang B, Yang J, Liu Y, Li X, Ma Z,, Lu P,, Liu Z,, Li J,. Dimeric c(RGD) peptide conjugated nanostructured lipid carriers for efficient delivery of Gambogic acid to breast cancer. Int J Nanomedicine. 2019 Aug 2;14:6179-6195. doi: 10.2147/IJN.S202424. eCollection 2019.

Lee PN, Ho WS. Antiproliferative activity of gambogic acid isolated from Garcinia hanburyi in Hep3B and Huh7 cancer cells. Oncol Rep. 2013 May;29(5):1744-50. doi: 10.3892/or.2013.2291. Epub 2013 Feb 19.

Li CY, Wang Q, Wang XM, Li GX, Shen S, Wei XL. Gambogic acid exhibits anti-metastatic activity on malignant melanoma mainly through inhibition of PI3K/Akt and ERK signaling pathways. Eur J Pharmacol. 2019 Dec 1;864:172719. doi: 10.1016/j.ejphar.2019.172719. Epub 2019 Oct 3.

Li R, Chen Y, Zeng LL, Shu WX, Zhao F, Wen L, Liu Y. Gambogic acid induces G0/G1 arrest and apoptosis involving inhibition of SRC-3 and inactivation of Akt pathway in K562 leukemia cells. Toxicology. 2009 Aug 3;262(2):98-105. doi: 10.1016/j.tox.2009.04.059. Epub 2009 May 9. 
Li X, Tang X, Su J, Xu G, Zhao L, Qi Q. Involvement of E-cadherin/AMPK/mTOR axis in LKB1induced sensitivity of non-small cell lung cancer to gambogic acid. Biochem Pharmacol. 2019 Nov;169:113635. doi: 10.1016/j.bcp.2019.113635. Epub 2019 Sep 9.

Lü L, Tang D, Wang L, Huang LQ, Jiang GS, Xiao XY, Zeng FQ. Gambogic acid inhibits TNF$\alpha$-induced invasion of human prostate cancer PC3 cells in vitro through PI3K/Akt and NF- $\mathrm{BB}$ signaling pathways. Acta Pharmacol Sin. 2012;33:531-541. doi: 10.1038/aps.2011.180.

Luo G, Jiang S, Zhang X, Ling Y, Luo H, Zhang Y. Gambogic Acid Affects Ribosomal Occurrence in Glioma Cells by Downregulating the Phosphoinositide Kinase-3/Protein Kinase B/Mammalian Target of Rapamycin Signaling Pathway. J Nanosci Nanotechnol. 2020 Jun 1;20(6):3361-3372. doi: 10.1166/jnn.2020.17425.

Luo GX, Cai J, Lin JZ, Luo WS, Luo HS, Jiang YY, Zhang Y. Autophagy inhibition promotes gambogic acid-induced suppression of growth and apoptosis in glioblastoma cells. Asian Pac J Cancer Prev. 2012;13(12):6211-6.

Mu R, Lu N, Wang J, Yin Y, Ding Y, Zhang X, Gui H, Sun Q, Duan H, Zhang L, Zhang Y, Ke $\mathrm{X}$, Guo Q. An oxidative analogue of gambogic acid-induced apoptosis of human hepatocellular carcinoma cell line HepG2 is involved in its anticancer activity in vitro. Eur J Cancer Prev. 2010 Jan;19(1):61-7. doi: 10.1097/CEJ.0b013e328333fb22.

Nie F, Zhang X, Qi Q, Yang L, Yang Y, Liu W, Lu N, Wu Z, You Q, Guo Q. Reactive oxygen species accumulation contributes to gambogic acid-induced apoptosis in human hepatoma SMMC-7721 cells. Toxicology. 2009 Jun 16;260(1-3):60-7. doi: 10.1016/j.tox.2009.03.010. Epub 2009 Mar 24.

Ortiz-Sánchez E, Daniels TR, Helguera G, Martinez-Maza O, Bonavida B, Penichet ML. Enhanced cytotoxicity of an anti-transferrin receptor IgG3-avidin fusion protein in combination with gambogic acid against human malignant hematopoietic cells: functional relevance of iron, the receptor, and reactive oxygen species. Leukemia. 2009 Jan;23(1):59-70. doi: 10.1038/leu.2008.270. Epub 2008 Oct 23.

Qiang L, Yang Y, You QD, Ma YJ, Yang L, Nie FF, Gu HY, Zhao L, Lu N, Qi Q, Liu W, Wang XT, Guo QL. Inhibition of glioblastoma growth and angiogenesis by gambogic acid: an in vitro and in vivo study. Biochem Pharmacol. 2008 Mar 1;75(5):1083-92. Epub 2007 Nov 9.

Saeed LM, Mahmood M, Pyrek SJ, Fahmi T, Xu Y, Mustafa T, Nima ZA, Bratton SM, Casciano D, Dervishi E, Radominska-Pandya A, Biris AS. Single-walled carbon nanotube and graphene 
nanodelivery of gambogic acid increases its cytotoxicity in breast and pancreatic cancer cells. $\mathrm{J}$ Appl Toxicol. 2014 Nov;34(11):1188-99. doi: 10.1002/jat.3018. Epub 2014 Sep 12.

Sang M, Han L, Luo R, Qu W, Zheng F, Zhang K, Liu F, Xue J, Liu W, Feng F. CD44 targeted redox-triggered self-assembly with magnetic enhanced EPR effects for effective amplification of gambogic acid to treat triple-negative breast cancer. Biomater Sci. 2019 Nov 1. doi: 10.1039/c9bm01171d. [Epub ahead of print]

Sang MM, Liu FL, Wang Y, Luo RJ, Huan XX, Han LF,, Zhang ZT, Feng F, Qu W, Liu $\mathrm{W}$, Zheng F,. A novel redox/pH dual-responsive and hyaluronic acid-decorated multifunctional magnetic complex micelle for targeted gambogic acid delivery for the treatment of triple negative breast cancer. Drug Deliv. $2018 \quad$ Nov;25(1):1846-1857. doi: 10.1080/10717544.2018.1486472.

Seo MJ,, Lee DM, Kim IY, Lee D, Choi MK, Lee JY, Park SS, Jeong SY, Choi EK, Choi KS,. Gambogic acid triggers vacuolization-associated cell death in cancer cells via disruption of thiol proteostasis. Cell Death Dis. 2019 Feb 22;10(3):187. doi: 10.1038/s41419-019-1360-4.

Shi X, Chen X, Li X, Lan X, Zhao C, Liu S, Huang H, Liu N, Liao S, Song W, Zhou P, Wang S, Xu L, Wang X, Dou QP, Liu J. Gambogic acid induces apoptosis in imatinib-resistant chronic myeloid leukemia cells via inducing proteasome inhibition and caspase-dependent Bcr-Abl downregulation. Clin Cancer Res. 2014 Jan 1;20(1):151-63. doi: 10.1158/1078-0432.CCR-131063. Epub 2013 Dec 12.

Shi X, Lan X, Chen X, Zhao C, Li X, Liu S, Huang H, Liu N, Zang D, Liao Y, Zhang P, Wang $\mathrm{X}$, Liu J. Gambogic acid induces apoptosis in diffuse large B-cell lymphoma cells via inducing proteasome inhibition. Sci Rep. 2015 Apr 8;5:9694. doi: 10.1038/srep09694.

Tao Z, Zhou Y, Lu J, Duan W, Qin Y, He X, Lin L, Ding J. Caspase-8 preferentially senses the apoptosis-inducing action of NG-18, a Gambogic acid derivative, in human leukemia HL-60 cells. Cancer Biol Ther. 2007 May;6(5):691-6. Epub 2007 Feb 3.

Thida M, Kim DW, Tran TTT, Pham MQ, Lee H, Kim I, Lee JW. Gambogic acid induces apoptotic cell death in T98G glioma cells. Bioorg Med Chem Lett. 2016 Feb 1;26(3):1097-1101. doi: 10.1016/j.bmcl.2015.11.043. Epub 2015 Nov 14.

Wang C, Zhang H, Chen B, Yin H, Wang W. Study of the enhanced anticancer efficacy of gambogic acid on Capan-1 pancreatic cancer cells when mediated via magnetic $\mathrm{Fe} 3 \mathrm{O} 4$ nanoparticles. Int J Nanomedicine. 2011;6:1929-35. doi: 10.2147/IJN.S24707. Epub 2011 Sep 9. 
Wang C, Zhang H, Chen Y, Shi F, Chen B. Gambogic acid-loaded magnetic Fe(3)O(4) nanoparticles inhibit Panc-1 pancreatic cancer cell proliferation and migration by inactivating transcription factor ETS1. Int J Nanomedicine. 2012;7:781-7. doi: 10.2147/IJN.S28509. Epub 2012 Feb 14.

Wang H\#, Zhao Z\#, Lei S, Li S, Xiang Z, Wang X, Huang X, Xia G, Huang X. Gambogic acid induces autophagy and combines synergistically with chloroquine to suppress pancreatic cancer by increasing the accumulation of reactive oxygen species. Cancer Cell Int. 2019 Jan 5;19:7. doi: 10.1186/s12935-018-0705-x. eCollection 2019.

Wang LH, Li Y, Yang SN, Wang FY, Hou Y, Cui W, Chen K, Cao Q, Wang S, Zhang TY, et al. Gambogic acid synergistically potentiates cisplatin-induced apoptosis in non-small-cell lung cancer through suppressing NF- $\mathrm{B}$ and MAPK/HO-1 signalling. Br J Cancer. 2014;110:341-352. doi: 10.1038/bjc.2013.752.

Wang Q, Wei J, Wang C, Zhang T, Huang D, Wei F, He F, Cai W, Yang P, Zeng S, Li W, Cao J. Gambogic acid reverses oxaliplatin resistance in colorectal cancer by increasing intracellular platinum levels. Oncol Lett. 2018 Aug;16(2):2366-2372. doi: 10.3892/ol.2018.8916. Epub 2018 Jun 6.

Wang S, Shao M, Zhong Z, Wang A, Cao J, Lu Y, Wang Y, Zhang J,. Co-delivery of gambogic acid and TRAIL plasmid by hyaluronic acid grafted PEI-PLGA nanoparticles for the treatment of triple negative breast cancer. Drug Deliv. 2017 Nov;24(1):1791-1800. doi: 10.1080/10717544.2017.1406558.

Wang S, Wang L, Chen M, Wang Y. Gambogic acid sensitizes resistant breast cancer cells to doxorubicin through inhibiting P-glycoprotein and suppressing survivin expression. Chem Biol Interact. 2015 Jun 25;235:76-84. doi: 10.1016/j.cbi.2015.03.017. Epub 2015 Mar 27.

Wang S, Xu Y, Li C, Tao H, Wang A, Sun C, Zhong Z, Wu X, Li P, Wang Y. Gambogic acid sensitizes breast cancer cells to TRAIL-induced apoptosis by promoting the crosstalk of extrinsic and intrinsic apoptotic signalings. Food Chem Toxicol. 2018b. Sep;119:334-341. doi: 10.1016/j.fct.2018.02.037. Epub 2018 Feb 16.

Wang S, Yang Y, Wang Y, Chen M. Gambogic acid-loaded $\mathrm{pH}$-sensitive mixed micelles for overcoming breast cancer resistance. Int J Pharm. 2015 Nov 30;495(2):840-8. doi: 10.1016/j.ijpharm.2015.09.041. Epub 2015 Sep 25. 
Wang X, Chen Y, Han QB, Chan CY, Wang H, Liu Z, Cheng CH, Yew DT, Lin MC, He ML, Xu HX, Sung JJ, Kung HF. Proteomic identification of molecular targets of gambogic acid: role of stathmin in hepatocellular carcinoma. Proteomics. $2009 \quad$ Jan;9(2):242-53. doi: 10.1002/pmic.200800155.

Wang Y, Liang X, Tong R, Yang J, Yang Y, Zhong J, Wu Q, Gong C, Yu J, Cai L. Gambogic Acid-Loaded Polymeric Micelles for Improved Therapeutic Effect in Breast Cancer. J Biomed Nanotechnol. 2018a. Oct 1;14(10):1695-1704. doi: 10.1166/jbn.2018.2626.

Wang Y, Sui Y, Tao Y. Gambogic acid increases the sensitivity to paclitaxel in drug-resistant triple-negative breast cancer via the SHH signaling pathway. Mol Med Rep. 2019 Nov;20(5):4515-4522. doi: 10.3892/mmr.2019.10697. Epub 2019 Sep 23.

Wei F, Zhang T, Yang Z, Wei JC, Shen HF, Xiao D, Wang Q, Yang P,, Chen HC, Hu H,, Chen ZP, Huang Q, Li WL, Cao J,. Gambogic Acid Efficiently Kills Stem-Like Colorectal Cancer Cells by Upregulating ZFP36 Expression. Cell Physiol Biochem. 2018;46(2):829-846. doi: 10.1159/000488740. Epub 2018 Mar 29.

Wei J, Yang P, Li W, He F, Zeng S, Zhang T, Zhong J, Huang D, Chen Z, Wang C, Chen H, Hu H, Cao J. Gambogic acid potentiates the chemosensitivity of colorectal cancer cells to 5fluorouracil by inhibiting proliferation and inducing apoptosis. Exp Ther Med. 2017 Feb;13(2):662-668. doi: 10.3892/etm.2017.4021. Epub 2017 Jan 2.

Wen C, Huang L, Chen J, Lin M, Li W, Lu B, Rutnam ZJ, Iwamoto A, Wang Z, Yang X, Liu H. Gambogic acid inhibits growth, induces apoptosis, and overcomes drug resistance in human colorectal cancer cells. Int J Oncol. 2015 Nov;47(5):1663-71. doi: 10.3892/ijo.2015.3166. Epub 2015 Sep 15.

Wu L, Guo H, Sun H, Zhang W, Sun C, Wang J. UNC119 mediates gambogic acid-induced cellcycle dysregulation through the Gsk $3 \beta / \beta$-catenin pathway in hepatocellular carcinoma cells. Anticancer Drugs. 2016 Nov;27(10):988-1000.

Xia G, Wang H, Song Z, Meng Q, Huang X, Huang X. Gambogic acid sensitizes gemcitabine efficacy in pancreatic cancer by reducing the expression of ribonucleotide reductase subunit-M2 (RRM2). J Exp Clin Cancer Res. 2017 Aug 10;36(1):107. doi: 10.1186/s13046-017-0579-0.

Xu J, Zhou M, Ouyang J, Wang J, Zhang Q, Xu Y, Xu Y, Zhang Q, Xu X, Zeng H. Gambogic acid induces mitochondria-dependent apoptosis by modulation of Bcl-2 and Bax in mantle 
cell lymphoma JeKo-1 cells. Chin J Cancer Res. $2013 \quad$ Apr;25(2):183-91. doi: 10.3978/j.issn.1000-9604.2013.02.06.

Xu P, Li J, Shi L, Selke M, Chen B, Wang X. Synergetic effect of functional cadmium-tellurium quantum dots conjugated with gambogic acid for HepG2 cell-labeling and proliferation inhibition. Int J Nanomedicine. 2013;8:3729-36. doi: 10.2147/IJN.S51622. Epub 2013 Sep 30.

Xu X, Liu Y, Wang L, He J, Zhang H, Chen X, Li Y, Yang J, Tao J. Gambogic acid induces apoptosis by regulating the expression of Bax and Bcl-2 and enhancing caspase- 3 activity in human malignant melanoma A375 cells. Int J Dermatol. 2009 Feb;48(2):186-92. doi: 10.1111/j.1365-4632.2009.03946.X.

Xu Y, Wang C, Ding Y, Wang Y, Liu K, Tian Y, Gao M, Li Z, Zhang J, Li L. Nanoparticles with Optimal Ratiometric Co-Delivery of Docetaxel with Gambogic Acid for Treatment of MultidrugResistant Breast Cancer. J Biomed Nanotechnol. 2016 Sep;12(9):1774-81.

Yan X, Yang Y, He L, Peng D, Yin D. Gambogic acid grafted low molecular weight heparin micelles for targeted treatment in a hepatocellular carcinoma model with an enhanced antiangiogenesis effect. Int J Pharm. 2017 Apr 30;522(1-2):110-118. doi: 10.1016/j.ijpharm.2017.02.051. Epub 2017 Feb 24.

Ye L, Zhou J, Zhao W, Jiao P, Ren G, Wang S. Gambogic acid-induced autophagy in nonsmall cell lung cancer NCI-H441 cells through a reactive oxygen species pathway. J Cancer Res Ther. 2018 Dec;14(Supplement):S942-S947. doi: 10.4103/0973-1482.206866.

Yin D, Yang Y, Cai H, Wang F, Peng D, He L. Gambogic acid-loaded electrosprayed particles for site-specific treatment of hepatocellular carcinoma. Mol Pharm. 2014 Nov 3;11(11):4107-17. doi: 10.1021/mp500214a. Epub 2014 Oct 7.

Youns M,,, ElKhoely A, Kamel R. The growth inhibitory effect of gambogic acid on pancreatic cancer cells. Naunyn Schmiedebergs Arch Pharmacol. 2018 May;391(5):551-560. doi: 10.1007/s00210-018-1485-5. Epub 2018 Mar 15.

Zhang H, Lei Y, Yuan P, Li L, Luo C, Gao R, Tian J, Feng Z, Nice EC, Sun J. ROS-mediated autophagy induced by dysregulation of lipid metabolism plays a protective role in colorectal cancer cells treated with gambogic acid. PLoS One. 2014 May 8;9(5):e96418. doi: 10.1371/journal.pone.0096418. eCollection 2014. 
Zhang Z, Qian H, Yang M, Li R, Hu J, Li L, Yu L, Liu B, Qian X. Gambogic acid-loaded biomimetic nanoparticles in colorectal cancer treatment. Int $\mathbf{J}$ Nanomedicine. 2017 Feb 28;12:1593-1605. doi: 10.2147/IJN.S127256. eCollection 2017.

Zhao T, Wang HJ, Zhao WW, Sun YL, Hu LK. Gambogic acid improves non-small cell lung cancer progression by inhibition of mTOR signaling pathway. Kaohsiung J Med Sci. 2017 Nov;33(11):543-549. doi: 10.1016/j.kjms.2017.06.013. Epub 2017 Jul 25.

Zhao Z, Zhang X, Wen L, Yi S, Hu J, Ruan J, Zhao F, Cui G, Fang J, Chen Y. Steroid receptor coactivator-3 is a pivotal target of gambogic acid in B-cell Non-Hodgkin lymphoma and an inducer of histone H3 deacetylation. Eur J Pharmacol. 2016 Oct 15;789:46-59. doi: 10.1016/j.ejphar.2016.06.048. Epub 2016 Jun 29.

Zhou Y, Wang R, Chen B, Sun D, Hu Y, Xu P. Daunorubicin and gambogic acid coloaded cysteamine-CdTe quantum dots minimizing the multidrug resistance of lymphoma in vitro and in vivo. Int J Nanomedicine. 2016 Oct 18;11:5429-5442. eCollection 2016.

Zhou Z, Ma J. Gambogic acid suppresses colon cancer cell activity in vitro. Exp Ther Med. 2019 Oct;18(4):2917-2923. doi: 10.3892/etm.2019.7912. Epub 2019 Aug 16.

Zhu M, Jiang Y, Wu H, Shi W, Lu G, Cong D, Liu K, Song S, Ren J. Gambogic Acid Shows AntiProliferative Effects on Non-Small Cell Lung Cancer (NSCLC) Cells by Activating Reactive Oxygen Species (ROS)-Induced Endoplasmic Reticulum (ER) Stress-Mediated Apoptosis. Med Sci Monit. 2019 May 29;25:3983-3988. doi: 10.12659/MSM.916835.

Zhu M, Wang M, Jiang Y, Wu H, Lu G, Shi W, Cong D, Song S, Liu K, Wang H. Gambogic Acid Induces Apoptosis of Non-Small Cell Lung Cancer (NSCLC) Cells by Suppressing Notch Signaling. Med Sci Monit. 2018 Oct 7;24:7146-7151. doi: 10.12659/MSM.912563. 